\title{
Validation of a green spectrophotometric method for the determination of dropropizine in commercial oral solutions
}

\author{
Millena Almeida Monsores ${ }^{\mathrm{a}}$, Mikaelly Pereira Caet ${ }^{\mathrm{a}}$, Anna Karolina Mouzer da Silva Machado ${ }^{\mathrm{b}}$, Marina Cardoso \\ Nemitz $^{a}$, Vítor Todeschinia ${ }^{a}$ Maximiliano da Silva Sangoi ${ }^{\mathrm{a}, \mathrm{b}, *}$ \\ ${ }^{a}$ Curso de Farmácia, UFRJ, Macaé-RJ, Brasil; ${ }^{b}$ Programa de Pós-graduação em Produtos Bioativos e Biociências, \\ UFRJ, Macaé-RJ, Brasil.
}

*Corresponding author: maxsilvasangoi@yahoo.com.br

The present work describes a green first-order derivative spectrophotometric (1D-UV) method for determination of dropropizine in commercial oral solutions. The method was developed using ecologically correct solvents and validated according to International Conference on Harmonization (ICH) recommendations. The response was linear in the concentration range of 6-24 $\mu \mathrm{g} / \mathrm{mL}(r=0.9997, n=7)$ at wavelength $249 \mathrm{~nm}$, which was the zero crossing point of excipient solutions. The detection and quantitation limits were 0.36 and $1.18 \mu \mathrm{g} / \mathrm{mL}$, respectively. The method showed adequate precision, with a relative standard deviation values lower than $1.41 \%$. Excellent values of accuracy were obtained, with a mean value of $99.44 \%$. The method proved to be robust by a full factorial design evaluation. It is simple, it has low cost, and it has low use of polluting reagents. Minimum environmental hazards observed and the results obtained attest to the reliability of the proposed green method, showing to be specific, linear, precise, accurate and robust. Thus, the validated 1D-UV spectrophotometric method was successfully applied to the quantitative analysis of dropropizine in oral solutions dosage forms, helping to improve quality control and environmental improvement.

Keywords: dropropizine; spectrophotometry; pharmaceutical analysis; validation; green method. https://doi.org/10.22456/2527-2616.108127

\section{Introduction}

The cough reflex serves to clear the airways of excessive secretions and foreign matter and can also be voluntarily evoked. It has a protective role, but can sometimes become excessive, non-productive and troublesome to patients. Cough is one of the most common reasons why individuals seek medical attention, and can be classified as acute cough (lasting less than 3-4 weeks) and chronic cough (lasting more than 8 weeks) $(1,2)$.

The most common cause of acute cough is an upper respiratory tract infection such as allergy, sinusitis, and flu. However, chronic cough can be a feature of a number of pulmonary disorders such as asthma, chronic obstructive pulmonary disease and lung cancer, or extrapulmonary disorders such as gastro-esophageal reflux disease $(1,2)$. Besides, dry-cough is one of the most common symptoms of coronavirus disease 2019 (COVID-19). COVID-19 is caused by severe acute respiratory syndrome coronavirus 2 (SARS-CoV-2). The SARS-CoV-2 primarily affects the respiratory system, and respiratory distress/failure is seen in severe/critically ill COVID-19 patients (3). Thus, the use of a cough suppressant can be applied in the palliative care setting. In patients, the cause of cough remains unexplained even after some low detailed assessments (4). Chronic cough has a prevalence of over $12 \%$ in the general population and can reduce the quality of life of patients and a significant economic cost for the individual and society $(1,2,5)$. There are very few safe and effective treatments for cough, consisting mainly of antitussives, expectorants and mucolytics, alone or in combinations $(6,7)$. The poor tolerability of most antitussives on the market is closely related to central nervous system side effects. However, dropropizine, a peripherally acting antitussive drug, has a good tolerability and safety profile (2).

Dropropizine (Figure 1) is chemically described as 3-(4phenyl-1-piperazinyl)-1,2-propanediol. This substance belongs to the class of organic compounds known as phenylpiperazines, which contains a phenylpiperazine skeleton, consisting of a piperazine bound to a phenyl group $(8,9)$. Dropropizine and its (S)-enantiomer, levodropropizine, act in non-productive cough of adults and children older than 2 years. The drugs inhibit the cough reflex through action on peripheral receptors and their afferent conductors $(1,10)$.

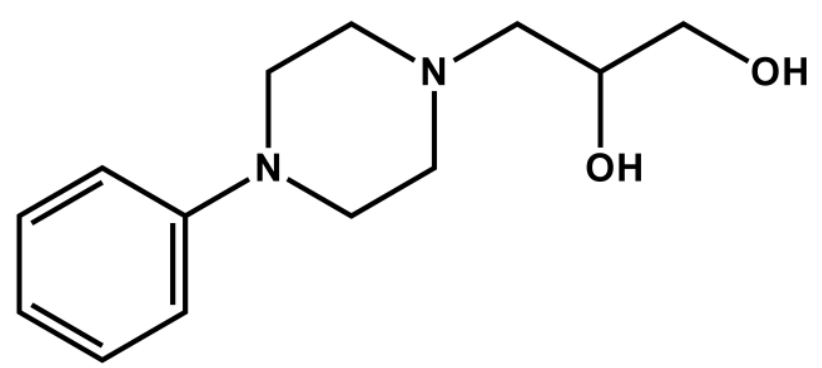

Figure 1. Chemical structure of dropropizine.

For routine analysis, a simple, rapid, and cost-effective analytical method is preferred. Spectrophotometric methods are the most commonly used techniques and continue to enjoy wide popularity. These methods are more economic and simpler, compared to methods such as chromatography and electrophoresis, and can provide 
a very useful alternative for routine analysis of pharmaceutical formulations (11-17). For comparison, one typical liquid chromatograph generates more than $1 \mathrm{~L}$ of organic waste daily. This amount of solvent waste is from the operation of liquid chromatographs only, without the waste originating from sample preparation (18).

Derivative spectrophotometry is an analytical technique which consists in the differentiating of normal spectrum by mathematical transformation of spectral curve into a derivative (first- or higher derivatives). This technique usually improves resolution bands, eliminates the influence of background or matrix and provides more defined fingerprints than traditional ordinary or direct absorbance spectra, since it enhances the detectability of minor spectral features (19). The common availability of the instrumentation, the simplicity of procedures, speed, precision and accuracy of the technique still make spectrophotometric methods attractive (20).

Green analytical chemistry is a research field that incorporates sustainable analytical approaches to minimize the toxicity and amounts of wastes keeping the analytical performance. There are many analytical strategies for this purpose, such as the miniaturization of the sample preparation, the application of solventless extraction techniques, the development of new technologies with low waste production and the substitution for less toxic solvents $(18,21-27)$.

Dropropizine is marketed in the form of a racemic mixture or its pure enantiomer, levodropropizine, and is available worldwide in various pharmaceutical forms such as tablets, syrup and oral solution. In Brazil, it is available only in liquid dosage forms of syrup and oral solution, in different concentrations. Due to the absence of analytical methods for the determination of this drug in oral solutions dosage form, the aim of this study was to develop and validate an analytical method with green spectrophotometric approaches, according to the International Conference on Harmonization (ICH) guideline (28). Therefore, this method contributes to the research on new analytical alternatives with advantages for the quality control of pharmaceutical formulations and, thus, promote public health and environmental benefits.

\section{Experimental}

\section{Chemicals and reagents}

Dropropizine reference standard was purchased from European Pharmacopoeia (Strasbourg, France). Dropropizine $30 \mathrm{mg} / \mathrm{mL}$ oral solutions were acquired on local market. All products were used within their shelf life period. All chemicals used were of pharmaceutical or special analytical grade. Ultrapure water (Milli Q Gradient System, Millipore Corp., Bedford, MA, USA) was used for all the analyses. Each oral liquid dosage form was labeled containing the following excipients: benzoic acid, citric acid, sodium benzoate, ethoxylated hydrogenated castor oil, sodium saccharin, sucralose, neroli aroma, purified water.

\section{Apparatus and experimental conditions}

A UV-Vis Lambda 35 double-beam spectrophotometer (PerkinElmer, Singapore) with $1 \mathrm{~cm}$ quartz cells was used. UV WinLab software 6.0 was used for instrument control and data acquisition. The first-order derivative spectra of solutions were recorded at a fast scan speed, the spectra were obtained by instrumental electronic differentiation using a wavelength interval of $2 \mathrm{~nm}$ in the range of 200-400 $\mathrm{nm}$. The determinations were made at $249 \mathrm{~nm}$. The spectrophotometric measurements were recorded by using water as a blank solution.

\section{Preparation of reference solution}

For the preparation of the reference solution, $30 \mathrm{mg}$ dropropizine reference substance was diluted in methanol, obtaining a concentration of $600 \mu \mathrm{g} / \mathrm{mL}$. The reference solution was stored at $2-8^{\circ} \mathrm{C}$ protected from light and diluted daily to an appropriate concentration with water.

\section{Preparation of sample solution}

A quantity equivalent of $30 \mathrm{mg}$ of dropropizine were transferred into individual $50 \mathrm{~mL}$ volumetric flasks. After adding $30 \mathrm{~mL}$ of methanol, the flasks were mixed for 3 minutes. The samples were made up to volume with the same diluent. This solution was filtered through a $0.45 \mu \mathrm{m}$ membrane filter (Millipore Corp). An aliquot of $125 \mu \mathrm{L}$ was transferred into a $5 \mathrm{~mL}$ volumetric flask and marked up to volume with water in order to produce a final concentration of $15 \mu \mathrm{g} / \mathrm{mL}$ (working solution).

\section{Validation of the 1D-UV method}

Method validation was performed following ICH specifications for specificity, linearity, accuracy, precision, robustness, limit of detection (LD) and quantitation (LQ) (28).

\section{Specificity}

The specificity evaluation was performed by preparing placebo containing the same excipients of the commercial products. Placebo solutions ( $15 \mu \mathrm{g} / \mathrm{mL}$ in theory) were prepared using the same procedure for the sample preparations $(n=3)$. In a separate study, drug with the same concentration was prepared independently from pure drug stock and analyzed. To maintain an adequate simulation of the presence of excipients in real samples, the concentration for both solutions was the same. All the solutions were scanned from 200 to $400 \mathrm{~nm}$, evaluated by first-derivative spectra and checked for any interference in the absorbance at tested wavelength $(249 \mathrm{~nm})$.

\section{Linearity}


The linearity was determined by constructing three independent analytical curves, each one with seven concentrations of dropropizine prepared in water, in the range of 6 to $24 \mu \mathrm{g} / \mathrm{mL}(6,9,12,15,18,21$, and 24 $\mu \mathrm{g} / \mathrm{mL})$. The linearity range was validated at $40-160 \%$ of the target assay concentration. The linearity was evaluated by linear regression analysis. The least-squares regression method was used to calculate the correlation coefficient, $y$-intercept, and slope of the regression line. Analysis of variance (ANOVA) was applied for proving compliance of the linear model.

\section{Precision}

The precision was determined by repeatability (intraday precision) and intermediate precision (interday precision). Repeatability was evaluated by assaying six determinations at the same concentration $(15 \mu \mathrm{g} / \mathrm{mL})$, during the same day, under the same experimental conditions. The intermediate precision was studied by comparing the results obtained on three different days. Precision was expressed as relative standard deviation (RSD).

\section{Accuracy}

This parameter was determined by the recovery test that consisted on adding known amounts of reference solution to the sample solution (prepared according to sample preparation). Aliquots of $50 \mu \mathrm{L}, 75 \mu \mathrm{L}$, and $100 \mu \mathrm{L}$ of dropropizine reference solution at a concentration of 600 $\mu \mathrm{g} / \mathrm{mL}$ were transferred to the sample solutions during the last dilution of the samples. The final concentrations of reference substance in each level were: 15,18 , and 21 $\mu \mathrm{g} / \mathrm{mL}$.

\section{$L D$ and $L Q$}

LD/LQ parameters are not a requirement for drug assay; however, it is always useful to demonstrate that the analyses are being conducted in a region which is above the LQ value. The LD and LQ were calculated based on the standard deviation of the response (y-intercepts of regression lines) and the slope using three independent analytical curves, as defined by ICH. LD and LQ were calculated as $3.3 \sigma / \mathrm{S}$ and $10 \sigma / \mathrm{S}$, respectively, where $\sigma$ is the standard deviation of the response and $\mathrm{S}$ is the slope of the calibration curve.

\section{Robustness}

In order to study the simultaneous variation of the factors in the considered responses, a multivariate approach using design of experiments is recommended in robustness testing. A full factorial design was used to examine the effects of three factors, studied at two levels (high and low): wavelength $(247 \mathrm{~nm} ; 251 \mathrm{~nm})$, different solvents (water: ethanol $(4: 1, \mathrm{v} / \mathrm{v})$; water: methanol $(4: 1, \mathrm{v} / \mathrm{v}))$ and sample temperature $\left(5{ }^{\circ} \mathrm{C} ; 23{ }^{\circ} \mathrm{C}\right)$ in 8 experiments. The low and high levels were deliberately selected on the basis of small changes in method parameters, providing an indication of its reliability during normal usage. The response is the percentage of dropropizine in the oral solutions dosage forms (relative to their label claimed concentration) obtained compared to the reference solution in each experiment. All experiments were performed in randomized order to minimize the effects of uncontrolled factors that may introduce a bias into the response. The statistical analyses of the data were performed by the Minitab 17 (Minitab Inc, State College, PA, USA) data analysis software.

\section{Sample analysis}

For dropropizine quantitation in the oral solutions dosage forms, the sample solutions prepared in triplicate were diluted to an appropriate concentration $(15 \mu \mathrm{g} / \mathrm{mL})$ with water, filtered, and the percentage recoveries of the drug calculated against the reference substance.

\section{Results and Discussion}

\section{Method development}

The spectrophotometric technique provides economic, practical and significant advantages over other methods. The development of the spectrophotometric method was more convenient, since it is simple, less time consuming and environmentally friendly for the routine analysis of dropropizine in oral solutions dosage forms. In addition, dropropizine syrup forms were also evaluated during development of the method, but due the presence of excipients that absorb light in the UV region, the method was not selective enough for syrup formulation.

The environmental impact of analytical procedures is the factor that has to be considered during solvent selection in optimization processes (18). In this work, different solvents were investigated to develop a suitable 1D-UV spectrophotometric method for the analysis of dropropizine in oral solutions. For selection of diluents, the criteria employed were the sensitivity of the method, the easiness of the sample preparation, and the solubility of the drug. Methanol was selected to first step dilution of dropropizine since it had a great capability to dissolve the drug in reference substance. However, in order to reduce the use of organic solvent, water was used as final diluent. Water is the better solvent considering toxicological risks and the absence of demanding residue storage, and it presented a good potential to dissolve the drug in conditions assayed. Considering sample preparation as usually the most polluting step in an analytical method, the use of non-polluting solvent was the ecological approach established in this work.

The use of solvents is one of the main environmental problems faced by analytical chemistry. Due to the impossibility of applying techniques with less waste production, solvents should be chosen with environmental awareness (18). There are solvent selection guides, developed by pharmaceutical companies that may give some clues for optimization methods in terms of solvents 
(29). All of these solvent selection guides are based on environmental, health and safety criteria. Alcohols and water, as used in this work, as well as some esters, are classified as "recommended". Hazardous or highly hazardous are hydrocarbons, chlorinated solvents and some of the ethers (18).

The drug stability study was accomplished for the development and validation of the method. The stability of dropropizine in water was evaluated to verify if any spontaneous degradation occurs when the samples are prepared. The data obtained showed that sample solutions were stable during up to $48 \mathrm{~h}$ at $2-8{ }^{\circ} \mathrm{C}$ and for $6 \mathrm{~h}$ at room temperature, showing nonsignificant change $(<2 \%)$ relative to freshly prepared samples, as suggested (30).

Zero-order UV-Vis spectrum of dropropizine in water showed maximum drug absorption wavelength around $237 \mathrm{~nm}$. However, an interference from the excipients present in oral solutions was verified in all the region of dropropizine absorption spectrum, which precludes the analytical use of zero-order spectrophotometry (Fig. 2a). The first derivative was considered to be ideal for solving the overlapping of excipients absorption over dropropizine signal. As observed in Figure $2 b$, the zero crossing for placebo solution appears at $249 \mathrm{~nm}$. Therefore, this value was selected as optimum to determine dropropizine in the presence of the pharmaceutical excipients.

Derivative spectra can be used to clarify absorption bands in more complex UV spectra. Compared with conventional spectrophotometric determinations, derivative spectrophotometry has proved to be of a great value in eliminating the interference from excipients (19), especially when applied to liquid formulations with large amounts of excipients with UV interference.
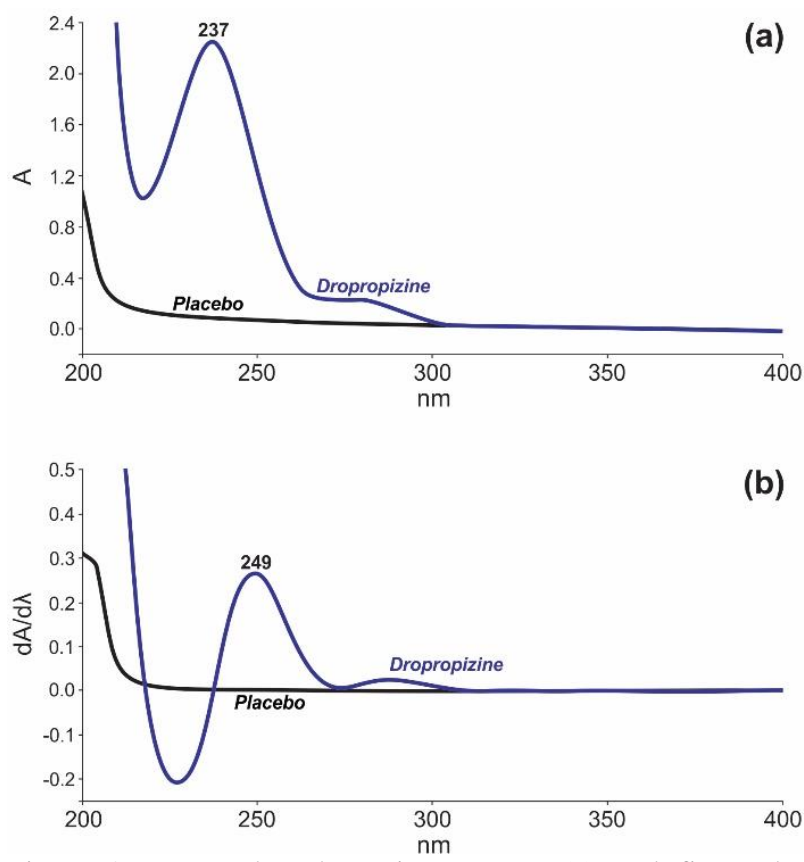

Figure 2. Zero-order absorption spectra (a) and first-order derivative spectra (b) of dropropizine reference substance solution and placebo solution, in water at concentration of 15 $\mu \mathrm{g} / \mathrm{mL}$.

\section{Method validation}

\section{Specificity}

The spectrum analyzes show that it was possible to identify the analyte in question (dropropizine) in the presence of the placebo solution (Fig. 2b). Both dropropizine and the placebo solution were diluted with water; the spectrum was successful in the first absorption order at $249 \mathrm{~nm}$, confirming the specificity of the method.

\section{Linearity}

A linear relationship was found between the absorbance and the concentration of dropropizine in the range of 6 to $24 \mu \mathrm{g} / \mathrm{mL}$ (Fig. 3). The representative linear equation was $y=0.0181 x+0.0007$, calculated by the least squares method, and the correlation coefficient ( $r=0.9997)$ was highly significant. The analytical data were validated by means of ANOVA that demonstrated significant linear regression $(f$ calculated $=21330.37>f$ critical $=4.60 ; P<$ $0.05)$ and no significant deviation from linearity $(f$ calculated $=2.48<f$ critical $=2.96 ; P>0.05)$. These results were proven according by successful application of the analytical method to synthetic mixtures of the drug product components to which known amount of analyte added within the range of method.

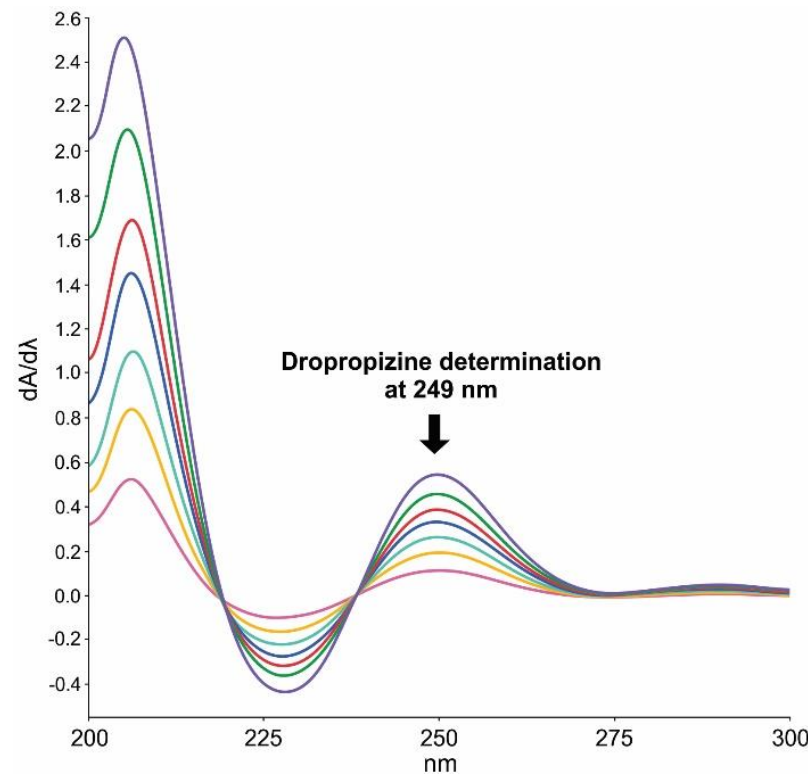

Figure 3. First order derivative spectra of dropropizine reference solutions, in water in the range of 6 to $24 \mu \mathrm{g} / \mathrm{mL}$.

\section{Precision}

Precision was determined by studying the intra-day and inter-day precision. The experimental values obtained for the determination of dropropizine in real samples are presented in Table 1 . The variability of the results was low with RSD values of less than $1.05 \%$ to intra-day precision, and the value of inter-day precision was $1.41 \%$ in oral solutions dosage forms. RSD values found for the analytical method were within the acceptable range 
indicating that this method has excellent repeatability and intermediate precision.

Table 1. Intra-day and inter-day precision data of 1D-UV spectrophotometric method for dropropizine oral solution dosage forms.

\begin{tabular}{ccc}
\hline Day & $\begin{array}{c}\text { Concentration found } \\
(\boldsymbol{\mu g} / \mathbf{m L})\end{array}$ & $\mathbf{R S D}^{\mathbf{2}(\boldsymbol{\%})}$ \\
\hline 1 & 15.04 & 0.99 \\
2 & 14.73 & 1.05 \\
3 & 15.09 & 0.83 \\
\hline Mean $^{3}$ & 14.96 & 1.41 \\
\hline
\end{tabular}

${ }^{1}$ Mean of six replicates, ${ }^{2} \mathrm{RSD}$ (Relative standard deviation), ${ }^{3}$ Mean of eighteen replicates.

\section{Accuracy}

The excellent mean percentage recovery values and their RSD values less than $2 \%$ were found satisfactory. At each level of the dropropizine concentration three determinations were performed. The mean recovery was $99.44 \%$ (RSD $=1.08 \%$ ) for oral solutions dosage forms (Table 2). These results revealed that any small change in drug concentration in these solutions could be accurately determined by the proposed analytical method.

Table 2. Experimental values obtained in the recovery test for dropropizine by using the 1D-UV spectrophotometric method.

\begin{tabular}{|c|c|c|c|c|}
\hline $\begin{array}{c}\text { Added } \\
\text { level } \\
(\mu \mathrm{g} / \mathrm{mL})\end{array}$ & $\begin{array}{c}\text { Nominal } \\
\text { concentration } \\
(\mu \mathrm{g} / \mathrm{mL})\end{array}$ & $\begin{array}{c}\text { Mean } \\
\text { concentration } \\
(\mu \mathrm{g} / \mathrm{mL})\end{array}$ & $\mathrm{RSD}^{2}$ & Accuracy \\
\hline 6 & 15 & 15.06 & $1.84 \%$ & $100.41 \%$ \\
\hline 9 & 18 & 17.93 & $1.63 \%$ & $99.62 \%$ \\
\hline 12 & 21 & 20.64 & $1.51 \%$ & $98.28 \%$ \\
\hline
\end{tabular}

${ }^{1}$ Mean of three replicates, ${ }^{2}$ RSD (Relative standard deviation).

\section{$L D$ and $L Q$}

For calculating the LD and LQ, the calibration equations were generated by using the mean values of the three independent standard curves. The obtained values were 0.36 and $1.18 \mu \mathrm{g} / \mathrm{mL}$, respectively.

\section{Robustness}

The susceptibility of the developed analytical methods to changes was tested in order to evaluate their robustness. For this purpose, a full factorial design was used; the experimental model and the corresponding responses are summarized in Table 3.

The significance of the effects was evaluated by a Pareto chart (Figure 4) that consists of bars with a length proportional to the absolute value of the estimated effect, divided by the pseudo-SE defined by Lenth (31). The codes $\mathrm{A}, \mathrm{B}$, and $\mathrm{C}$ correspond to each of the parameters. The combination of two or three codes indicates the interaction effect between the variables. The bars are displayed based in the size of the effect, with the largest effect on the top. The chart includes a vertical line at the critical t-value for an $\alpha$ value of 0.05 . The effects in which the bars are smaller than the critical t-value were not considered significant and did not affect the response variables (32).

Table 3. Selected full factorial design for the robustness testing of dropropizine.

\begin{tabular}{ccccc}
\hline Assay & $\begin{array}{c}\text { Wavelength } \\
(\mathbf{n m})\end{array}$ & $\begin{array}{c}\text { Temperature } \\
\left({ }^{\circ} \mathbf{C}\right)\end{array}$ & Solvent $^{1}$ & $\begin{array}{c}\text { Assay }^{2} \\
(\%)\end{array}$ \\
\hline 1 & 251 & 23 & A & $97.30 \%$ \\
2 & 251 & 23 & B & $97.11 \%$ \\
3 & 251 & 5 & B & $98.83 \%$ \\
4 & 247 & 5 & A & $98.33 \%$ \\
5 & 247 & 5 & B & $99.95 \%$ \\
6 & 247 & 23 & A & $101.47 \%$ \\
7 & 247 & 23 & B & $100.85 \%$ \\
8 & 251 & 5 & A & $99.51 \%$ \\
\hline
\end{tabular}

${ }^{1}$ Solvent A (water: methanol, 4:1); Solvent B (water: ethanol, $4: 1),{ }^{2}$ Mean of three replicates.

\section{Method Application}

Acceptable results of application of the proposed method in oral solution dosage forms (Table 4) were obtained, demonstrating the quality of the pharmaceutical samples and the applicability of the validated method for quality control analysis of dropropizine. It should be considered that excipients did not interfere in the determination of the active compound. In addition, it is important to note that the method is not selective for the determination of enantiomeric purity and can be successfully applied in the analysis of dropropizine or levodropropizine oral solutions.

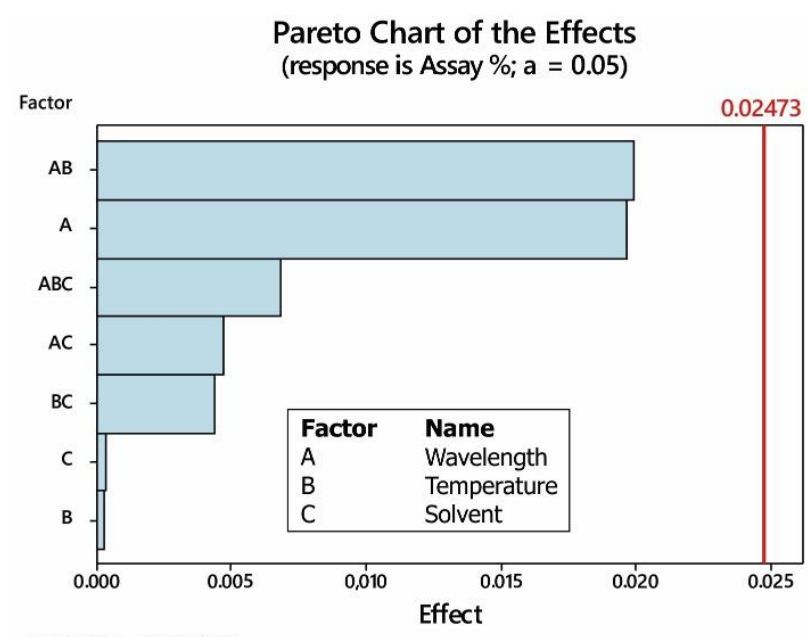

Lenth's PSE $=0.00656977$

Figure 4. Pareto charts representing the effects of the variables and their interactions on the dropropizine assay for the robustness test using the full factorial design.

\section{Conclusions}

Producing drugs with proven safety, quality, and efficacy should be a concern of all pharmaceutical industries. In 
this sense, development of appropriate analytical methods is necessary for the quality control of pharmaceuticals. This work presents an environmentally friendly, useful, simple, efficient and reliable 1D-UV spectrophotometric method for the determination of dropropizine in oral solution dosage forms requiring only minor sample preparation steps. The green method was validated showing satisfactory data for all tested parameters, thus offering advantages over other analytical methods due to its speed, simplicity, and lower cost. Besides, the method produces very low levels of hazardous waste, promoting benefits to public health and the environment and contributing to the technological and scientific domain in the area of quality control of pharmaceutical products. Therefore, the proposed method is adequate and can be conveniently used for the routine quality control of dropropizine in oral solutions.

\section{Acknowledgements}

The authors wish to thank CNPq, FAPERJ and PIBICUFRJ for the financial support.

\section{Conflict of interest}

The authors declare no conflicts of interest.

\section{References}

1. Dicpinigaitis PV, Morice AH, Birring SS, McGarvey L, Smith JA, Canning BJ, et al. Antitussive drugs - Past, present, and future. Pharmacol Rev. 2014; 66: 1-44.

2. Birring S, de Blasio F, Dicpinigaitis PV, Fontana G, Lanata L, Page C, et al. Antitussive therapy: A role for levodropropizine. Pulm Pharmacol Ther. 2019; 56: 7985 .

3. Rahman A, Niloofa R, De Zoysa IM, Cooray AD, Kariyawasam J, Seneviratne SL. Neurological manifestations in COVID-19: A narrative review. SAGE Open Med. 2020; 8: 1-10.

4. Haque RA, Usmani OS, Barnes PJ. Chronic idiopathic cough: a discrete clinical entity? Chest. 2005; 127(5): 1710-1713.

5. Morice AH, Fontana GA, Belvisi MG, Birring SS, Chung KF, Dicpinigaitis PV. ERS guidelines on the assessment of cough. Eur Respir J. 2007; 29: 12561276.

6. Chung KF, Chang AB. Therapy for cough: active agents. Pulm Pharmacol Ther. 2002; 15: 335-338.

7. Balbani AP. Cough: neurophysiology, methods of research, pharmacological therapy and phonoaudiology. Int Arch Otorhinolaryngol. 2012; 16: 259-268.
8. Drug Bank. Drug \& Drug Target Database.

9.European Pharmacopoeia. 8th. Edition. Strasbourg: Council of Europe, 2013.

10. Dicpinigaitis PV. Current and future peripherallyacting antitussives. Respir Physiol Neuro. 2006; 152: 356-362.

11. Sangoi MS, Todeschini V, Steppe M. Second-order derivative UV spectrophotometric method for the determination of fesoterodine and comparison with LC, CE and LC-MS/MS in commercial extendedrelease tablets. Acta Chim Slov. 2012; 59: 136-143.

12. Ashour S, Bayram R. Selective and validated kinetic spectrophotometric method for the determination of irbesartan in pure and pharmaceutical formulations. Ann Pharm Fr. 2019; 77(2): 101-111.

13. Corte AC, Sfair, LL. Development and validation of analytical methodology for quality evaluation of tibolone in capsule pharmaceutical form through UV spectrophotometry. Drug Analytical Research. 2019; 3(2):7-11.

14. Gomes P, Negretto CMU, Naisinger ZB, Lorenzoni R, Wingert NR, Raffin RP. Second-derivative spectrophotometry for the analysis of simvastatin in polymeric Nanocapsules. Drug Anal Res. 2019; 3(2): 12-17.

15. Oppe TP, Menegola J, Schapoval EES. Development and validation of UV spectrophotometry and liquid chromatography methods for determination of cefpirome in raw material and pharmaceutical dosage. Drug Anal Res. 2019; 3(1): 42-50.

16. Naguib AI, Abdelaleem EA, Hassan ES, Hassan ES. Comparative study of eco-friendly spectrophotometric methods for accurate quantification of mebendazole and quinfamide combination; Content uniformity evaluation. Spectrochim Acta A. 2020; 235: 1-10.

17. Darwish HW, Ali NA, Naguib IA, Ghobashy MRE, Al-Hossaini AM, Abdelrahman MM. Stability indicating spectrophotometric methods for quantitative determination of bromazepam and its degradation product. Spectrochim Acta A. 2020; 238: $1-10$.

18. Tobiszewski M. Metrics for green analytical chemistry. Anal Methods. 2016; 8: 2993-2999.

19. Ojeda CB, Rojas FS. Recent developments in derivative ultraviolet/visible absorption spectrophotometry. Anal Chim Acta. 2004; 518(1-2): $1-24$. 
20. Rojas FS, Ojeda CB. Recent development in derivative ultraviolet/visible absorption spectrophotometry: 2004-2008: a review. Anal Chim Acta. 2009; 635(1): 22-44.

21. Anastas PT. Green chemistry and the role of analytical methodology development. Crit Rev Anal Chem. 1999; 29:3, 167-175.

22. Keith LH, Gron LU, Young JL. Green analytical methodologies. Chem Rev. 2007; 107: 2695-2708.

23. Mohamed HM. Green, environment-friendly, analytical tools give insights in pharmaceuticals and cosmetics analysis. Trends Anal Chem. 2015; 66: 176192.

24. Korany MA, Mahgoub H, Haggag RS, Ragab MAA, Elmallah OAA. Green chemistry: Analytical and chromatography. J Liq Chromatogr Relat Technol. 2017; 40(16): 839-852.

25. Saroj S, Shah P, Jairaj V, Rathod R. Green analytical chemistry and quality by design: A combined approach towards robust and sustainable modern analysis. Curr Anal Chem. 2018; 14(4): 367-381.

26. Gama MR, Melchert WR, Paixão TRLC, Rocha FRP. An overview of the Brazilian contributions to green analytical chemistry. An Braz Acad Sci. 2019; 91: 133.

27. Merey HA, Ramadan NK, Diab SS, Moustafa AA. Green spectrophotometric methods for the determination of a binary mixture of lidocaine hydrochloride and cetylpyridinium chloride in the presence of dimethylaniline. Spectrochim Acta A Mol Biomol Spectrosc. 2020; 242: 1-12.

28. International Conference on Harmonization (ICH) of Technical Requirements for the Registration of Pharmaceutical for Human Use. Validation of Analytical Procedures: Text and Methodology Q2(R1). Geneva, Switzerland, 2005. 17p.

29. Prat D, Hayler J, Wells A. A Survey of solvent selection guides. Green Chem. 2014; 16: 4546-4551.

30. Shabir GA. Validation of high-performance liquid chromatography methods for pharmaceutical analysis. Understanding the differences and similarities between validation requirements of the US Food and Drug Administration, the US Pharmacopeia and the International Conference on Harmonization. 2003; 987(1-2): 57-66

31. Lenth RV. Quick and easy analysis of unreplicated factorials. Technometrics. 1989; 31: 469-473.
32. Vanaja K, Rani RHS. Design of experiments: concept and applications of Plackett Burman design. Clin Res Regul Aff. 2008; 24(1): 1-23. 